\title{
On Generally Covariant Quantum Field Theory and Generalized Causal and Dynamical Structures
}

\author{
Ulrich Bannier
}

II. Institut für Theoretische Physik der Universität Hamburg, Luruper Chaussee 149, D-2000 Hamburg 50, Federal Republic of Germany

\begin{abstract}
We give an example of a generally covariant quasilocal algebra associated with the massive free field. Maximal, two-sided ideals of this algebra are algebraic representatives of external metric fields. In some sense, this algebra may be regarded as a concrete realization of Ekstein's ideas of presymmetry in quantum field theory. Using ideas from our example and from usual algebraic quantum field theory, we discuss a generalized scheme, in which maximal ideals are viewed as algebraic representatives of dynamical equations or Lagrangians. The considered frame is no quantum gravity, but may lead to further insight into the relation between quantum theory and space-time geometry.
\end{abstract}

\section{Introduction}

One of the most fascinating challenges of contemporary physics is the unification of Einstein's general relativity theory with quantum theory. Many attempts are made, but the goal seems to be still far off. It even is not clear how much of the conceptual and technical structures of both theories will survive an unification, because they seem to be fundamentally different.

By results of $[1,3,5,7$, and 8$]$, there emerge ideas of a quantum field theory which incorporates at least one of the basic principles of general relativity theory: the principle of general covariance. The setting is algebraic quantum field theory which seems to be especially suitable.

In algebraic quantum field theory (cf. Haag and Kastler [10]) to each region (open set with compact closure) $\mathcal{O}$ of Minkowski space $\mathbb{M}$ there corresponds one $C^{*}$-algebra $\mathscr{A}(\mathcal{C})$. This correspondence is assumed to fullfill the isotony property, i.e., if $\mathcal{O}_{1} \subseteq \mathcal{O}_{2} \subseteq \mathbb{M}$, then $\mathscr{A}\left(\mathcal{O}_{1}\right) \subseteq \mathscr{A}\left(\mathcal{O}_{2}\right)$. The self-adjoint elements of $\mathscr{A}(\mathcal{O})$ are interpreted as observables that detect events within $\mathcal{O}$. All $\mathscr{A}(\mathcal{O})$ are subalgebras of a $C^{*}$-algebra $\mathscr{A}$ which is the inductive limit of all the $\mathscr{A}(\mathcal{O}) . \mathscr{A}$ is also called algebra of quasilocal observables, and the correspondence $\mathbb{C} \rightarrow \mathscr{A}(\mathcal{O})$ net of local $C^{*}$-algebras. Among the (Haag-Kastler) axioms of algebraic quantum field theory 
there is one group which relates the causal structure and symmetry of space-time with the algebras:

\section{Causality}

(a) (Sometimes called Einstein causality.) If two regions $\mathcal{O}_{1}, \mathcal{O}_{2}$ lie space-like to each other, then the elements of $\mathscr{A}\left(\mathcal{O}_{1}\right)$ commute with those of $\mathscr{A}\left(\mathcal{O}_{2}\right)$, i.e. $\left[\mathscr{A}\left(\mathcal{O}_{1}\right), \mathscr{A}\left(\mathcal{O}_{2}\right)\right]=\{0\}$.

(b) (Sometimes calles primitive causality, which expresses the existence of a causal dynamical law.) If $\mathcal{O}_{2}$ is in the domain of dependence of $\mathcal{O}_{1}$ (every endless, nowhere space-like, smoth curve through $\mathcal{O}_{2}$ intersects $\left.\mathcal{O}_{1}\right)$, then $\mathscr{A}\left(\mathcal{O}_{2}\right) \subseteq \mathscr{A}\left(\mathcal{O}_{1}\right)$.

\section{Poincaré Invariance}

The orthochronuous Poincare group $\mathscr{P}_{+}^{\uparrow}$ is represented by automorphisms $\alpha_{(a, \Lambda)}$, acting on $\mathscr{A}$ by $\alpha_{(a, \Lambda)}(\mathscr{A}(\mathcal{O}))=\mathscr{A}(a+\mathscr{A} \mathcal{O}),(a, \Lambda) \in \mathscr{P}_{+}^{\uparrow}$. Here, $a$ is a translation vector and $\Lambda$ a Lorentz transformation.

In his Gibbs lecture Missed Opportunities Dyson [6] proposed the creation of a generally covariant formulation of these axioms, including some analog of a metric tensor in order to give a meaning to space-like separation. It is our opinion that one can fullfill Dyson's demand. In the next section we will give an example of a generally covariant net of $C^{*}$-algebras and generalized causal relations. The main additional structure we need, is the presence of many maximal, two-sided ideals in the local algebras $\mathscr{A}(\mathcal{C})$, by which we can formulate dynamical or causal structures. In algebraic quantum field theory the algebra of observables is usually assumed to be simple.

\section{Generally Covariant Weyl Algebra}

For clarity and simplicity, in the following example the space-time manifold is taken to be $\mathbb{R}^{4}$. The set of all globally hyperbolic metric tensor fields $\mathbf{g}$ on $\mathbb{R}^{4}$ is denoted by $\mathscr{G}$. By these prerequisites all of the following has a global character, but there are no fundamental obstacles to find a local formulation for the derived result.

In an interesting paper, Dimock [3] constructed to a given manifold with a globally hyperbolic metric g a Weyl algebra $\mathscr{A}_{\mathrm{g}}$ and a net $\mathcal{O} \rightarrow \mathscr{A}_{\mathrm{g}}(\mathcal{O})$ of $C^{*}$-subalgebras. We give a brief description of this net (using a different method than Dimock), since we shall need it afterwards for our construction of the generally covariant net. If $\Phi_{\mathbf{g}}$ denotes the quantum field and $f \in \mathscr{D}$ is a test function $(\mathscr{D}$ denotes the class of infinitely often differentiable functions with compact support in $\mathbb{R}^{4}$ ), the Weyl algebra $\mathscr{A}_{\mathbf{g}}$ is generated by elements $\exp i \Phi_{\mathbf{g}}(f)$, where in the usual field theoretic notation

$$
\Phi_{\mathbf{g}}(f)=\int \Phi_{\mathbf{g}}(x) f(x) d \mu_{\mathbf{g}}(x), \quad d \mu_{\mathbf{g}}(x)=\sqrt{-\operatorname{det} \mathbf{g}(x)} d x,
$$

and $\Phi \mathbf{g}$ satisfies the Klein-Gordon equation

$$
\left(\square_{\mathbf{g}}-m^{2}\right) \Phi_{\mathbf{g}}=0, \quad \square_{\mathbf{g}}=\frac{1}{\sqrt{-\operatorname{det} \mathbf{g}}} \partial_{\mu} g^{\mu v} \sqrt{-\operatorname{det} \mathbf{g} \partial_{v}} .
$$


Due to Eq. (2.1) the map $f \rightarrow \Phi_{\mathbf{g}}(f)$ is many to one

$$
\Phi_{\mathbf{g}}(f)=0 \Leftrightarrow f \in \mathbf{K}_{\mathbf{g}}:=\left\{f \in \mathscr{D} \mid G_{\mathbf{g}} f \equiv 0\right\},
$$

where $G_{\mathbf{g}}(x, y)=G_{\mathbf{g}}^{\mathrm{ret}}(x, y)-G_{\mathbf{g}}^{\mathrm{adv}}(x, y)$ is the causal Green's function of the KleinGordon operator, uniquely determined by the globally hyperbolic metric $\mathbf{g}$ (cf. [9]), and

$$
\left(G_{\mathbf{g}} f\right)(x)=\int_{\mathbb{R}^{4}} G_{\mathbf{g}}(x, y) f(y) d \mu_{\mathbf{g}}(y) .
$$

For $G_{\mathbf{g}}(x, y)$ we have $G_{\mathbf{g}}(x, y)=-G_{\mathbf{g}}(y, x)$, and $G_{\mathrm{g}} f$ is a solution of (2.1). Furthermore

$$
\operatorname{supp} G_{\mathbf{g}} f \cong J(\operatorname{supp} f) \quad \forall f \in \mathscr{D} .
$$

Here $J(\operatorname{supp} f)$ is the set of all points of $\mathbb{R}^{4}$ which can be reached by a causal curve through the support of $f$.

We can define the factor space $\mathbf{C}_{\mathbf{g}}:=\mathscr{D} / \mathbf{K}_{\mathbf{g}}$, and denote by $f^{\mathbf{g}}$ the element of $\mathbf{C}_{\mathbf{g}}$ which corresponds to the class of the element $f \in \mathscr{Z}$. Then we have the nondegenerate symplectic form

on $\mathbf{C}_{\mathbf{g}}$.

$$
\sigma_{\mathbf{g}}\left(f^{\mathbf{g}}, h^{\mathbf{g}}\right):=\int_{\mathbb{R}^{4}} f(x) G_{\mathbf{g}}(x, y) h(y) d \mu_{\mathbf{g}}(x) d \mu_{\mathbf{g}}(y), \quad f \in f^{\mathbf{g}}, h \in h^{\mathbf{g}},
$$

For the construction of the algebras $\mathscr{A}_{\mathbf{g}}$ we assign an abstract element $W_{\mathbf{g}}\left(f^{\mathbf{g}}\right)$ to every $f^{\mathbf{g}} \in \mathbf{C}_{\mathbf{g}}$, take all finite linear combinations, and define a star operation (involution) by $W_{\mathbf{g}}^{*}\left(f^{\mathbf{g}}\right):=W_{\mathbf{g}}\left(-f^{\mathbf{g}}\right)$ and a multiplication by the Weyl relation

$$
W_{\mathbf{g}}\left(f_{1}^{\mathbf{g}}\right) W_{\mathbf{g}}\left(f_{2}^{\mathbf{g}}\right):=e^{-\frac{i}{2} \sigma_{\mathbf{g}\left(f_{1}^{\mathrm{g}}, f_{2}^{\mathrm{g}}\right)}} W_{\mathbf{g}}\left(f_{1}^{\mathbf{g}}+f_{2}^{\mathbf{g}}\right) .
$$

The set of all these expressions forms a ${ }^{*}$-algebra $\tilde{\mathscr{A}}_{\mathbf{g}}$ with $W_{\mathrm{g}}\left(0^{\mathbf{g}}\right)$ as unit element. On $\tilde{\mathscr{A}}_{\mathrm{g}}$ we can define a (unique) $C^{*}$-norm, most simply by GNS-construction, starting from the positive linear form $\omega$ ) (cf. [2, p. 79]) defined by

$$
\omega\left(W_{\mathbf{g}}\left(f^{\mathbf{g}}\right)\right):= \begin{cases}1 & \text { if } f^{\mathbf{g}}=0^{\mathbf{g}} \\ 0 & \text { otherwise } .\end{cases}
$$

The completion of $\tilde{\mathscr{A}}_{\mathrm{g}}$ in this norm gives a unique and simple Weyl algebra $\mathscr{A}_{\mathrm{g}}$ (cf. [2, p. 20]). The net structure is obtained by assigning to the region $\mathcal{O}$ the subspace $\mathbf{C}_{\mathbf{g}}(\mathcal{O})$ of all classes $f^{\mathrm{g}}$ which possess a representative in $\mathscr{D}$ with support in $\mathcal{O} \cdot \mathscr{A}_{\mathrm{g}}(\mathcal{O})$ is then defined as the Weyl algebra generated from this subspace.

We now have for every metric field $\mathbf{g} \in \mathscr{G}$ a net $\mathcal{O} \rightarrow \mathscr{A}_{\mathbf{g}}(\mathcal{O})$ of $C^{*}$-algebras, which we need in the construction of a generally covariant net of $C^{*}$-algebras. The motivation for the construction is given by the following heuristic consideration.

A physical measurement will be performed within three steps:

(1) The measurement will be planned and described.

(2) The detector for the measurement will be put up in the presence of some boundary conditions (e.g. the existence of a gravitational field).

(3) The measurement will be carried out with a state.

We can take the point of view that, for fixed $f \in \mathscr{D}$, the elements $W_{\mathbf{g}_{1}}\left(f^{\mathbf{g}_{1}}\right)$ and $W_{\mathbf{g}_{2}}\left(f^{\mathbf{g}_{2}}\right)$, belonging to different algebras $\mathscr{A}_{\mathbf{g}_{1}}$ and $\mathscr{A}_{\mathbf{g}_{2}}$, represent the same 
description of a measurement or (for non-self-adjoint elements) operation procedure, differing in the circumstances under which they were carried out. Therefore, for fixed $f \in \mathscr{D}$, the set $\left\{W_{\mathbf{g}}\left(f^{\mathbf{g}}\right) \mid \mathbf{g} \in \mathscr{G}\right\}$ abstracts in a natural way from the metric field $\mathbf{g}$ and can be regarded as the mathematical image of the description of an operation procedure. Instead of those sets we regard the mappings

$$
W(f): \mathbf{g} \rightarrow W_{\mathbf{g}}\left(f^{\mathbf{g}}\right), \quad f \in \mathscr{D}, \quad \mathbf{g} \in \mathscr{G}, \quad W_{\mathbf{g}}\left(f^{\mathbf{g}}\right) \in \bigcup_{\mathbf{g} \in \mathscr{G}} \mathscr{A}_{\mathbf{g}},
$$

with $W(f)(\mathscr{G})$ equal to the mentioned set $\left\{W_{\mathbf{g}}\left(f^{\mathbf{g}}\right) \mid \mathbf{g} \in \mathscr{G}\right\}$. The mappings $W(f)$ may be viewed (without regarding a topology) as special sections of a bundle with base $\mathscr{G}$ and fibers $\mathscr{A}_{\mathrm{g}}$. They generate a ${ }^{*}$-algebra $\tilde{\mathscr{A}}$ by pointwise operations. The adjoint of $W(f)$ is defined by $W^{*}(f): \mathbf{g} \rightarrow W_{\mathrm{g}}\left(-f^{\mathbf{g}}\right)$. For a general section $A \in \tilde{\mathscr{A}}$ we write $A: \mathbf{g} \rightarrow A_{\mathrm{g}}$. In $\tilde{\mathscr{A}}$ we have the relation (flexible Weyl relation)

$$
W(f) W(h)=e^{-\frac{1}{2} \sigma(f, h)} W(f+h)
$$

with

$$
\sigma(f, h): \mathbf{g} \rightarrow \sigma_{\mathbf{g}}\left(f^{\mathbf{g}}, h^{\mathbf{g}}\right) .
$$

By the norms \|\|$_{\mathrm{g}}$ of all the $\mathscr{A}_{\mathrm{g}}$ we define by

$$
p_{\mathbf{g}}(A):=\left\|A_{\mathbf{g}}\right\|_{\mathbf{g}}
$$

$C^{*}$-seminorms, and by

$$
\|A\|:=\sup _{\mathbf{g} \in \mathscr{G}} p_{\mathrm{g}}(A)
$$

a $C^{*}$-norm on $\tilde{\mathscr{A}}$. With help of (2.4) it is easily verified that the supremum exists. The completion of $\tilde{\mathscr{A}}$ in this norm is a $C^{*}$-algebra $\mathscr{A}$. For a region $\mathbb{C} \subset \mathbb{R}^{4}, \mathscr{A}(\mathcal{O})$ is the smallest $C^{*}$-subalgebra of $\mathscr{A}$ which contains all those $W(f)$ with supp $f \subseteq \mathcal{O}$.

Every $C^{*}$-seminorm $p_{\mathrm{g}}$ defines a two-sided ideal in $\mathscr{A}$ by $\mathscr{T}_{\mathrm{g}}:=\operatorname{ker} p_{\mathrm{g}}$ which is closed in norm (cf. [4, p. 24]). We easily conclude that in the sense of isomorphy

and

$$
\mathscr{A} / \mathscr{T}_{\mathrm{g}}=\mathscr{A}_{\mathrm{g}}
$$

$$
\mathscr{A}(\mathcal{O}) /\left(\mathscr{A}(\mathcal{O}) \cap \mathscr{T}_{\mathrm{g}}\right)=\left(\mathscr{A}(\mathcal{O})+\mathscr{T}_{\mathrm{g}}\right) / \mathscr{T}_{\mathrm{g}}=\mathscr{A}_{\mathrm{g}}(\mathcal{O}) .
$$

(For the first isomorphy of (2.6) compare [4, p. 22].) As all $\mathscr{A}_{\mathrm{g}}$ are simple, the ideals $\mathscr{T}_{\mathrm{g}}$ are maximal in $\mathscr{A}$.

We want to mark that by the above process of idealization, the elements $A$ of $\mathscr{A}$ abstract from the external conditions, and our scheme is, therefore, closely related to Ekstein's [7] discussion on presymmetry where he also considered algebras with many ideals.

For the obtained net $\mathcal{O} \rightarrow \mathscr{A}(\mathcal{O})$, the causality axiom $I$ generalizes to

Theorem 2.1. Flexible Causality.

(a) If two regions $\mathcal{O}_{1}$ and $\mathscr{O}_{2}$ of $\mathbb{R}^{4}$ lie space-like to each other with respect to $\mathbf{g} \in \mathscr{G}$, then $\left[\mathscr{A}\left(\mathcal{O}_{1}\right), \mathscr{A}\left(\mathbb{O}_{2}\right)\right] \cong \mathscr{T}_{\mathbf{g}}$.

(b) If $\mathscr{O}_{1}, \mathcal{O}_{2} \subset \mathbb{R}^{4}$ are two regions and $\mathcal{O}_{2}$ is in the domain of dependence of $\mathscr{O}_{1}$ with respect to $\mathbf{g} \in \mathscr{G}$, then $\mathscr{A}\left(\mathbb{O}_{2}\right) \subseteq \mathscr{A}\left(\mathbb{O}_{1}\right)+\mathscr{T}_{\mathbf{g}}$. 
Proof. a) By virtue of (2.5) and (2.6) we have

$$
\left[\mathscr{A}\left(\mathscr{O}_{1}\right), \mathscr{A}\left(\mathcal{O}_{2}\right)\right] \subseteq \mathscr{T}_{\mathbf{g}} \Leftrightarrow\left[\mathscr{A}_{\mathbf{g}}\left(\mathcal{O}_{1}\right), \mathscr{A}_{\mathbf{g}}\left(\mathscr{O}_{2}\right)\right]=\{0\} .
$$

If two test functions $f_{1}, f_{2} \in \mathscr{D}$ fullfill $\operatorname{supp} f_{1} \subseteq \mathcal{O}_{1}$ and $\operatorname{supp} f_{2} \subseteq \mathcal{O}_{2}$, then we conclude with help of $(2.2) \sigma_{\mathbf{g}}\left(f_{1}^{\mathbf{g}}, f_{2}^{\mathbf{g}}\right)=0$. With this result and the Weyl relation we get

$$
\left[W_{\mathbf{g}}\left(f_{1}^{\mathbf{g}}\right), W_{\mathbf{g}}\left(f_{2}^{\mathbf{g}}\right)\right]=0 \quad \forall W_{\mathbf{g}}\left(f_{i}^{\mathbf{g}}\right) \in \mathscr{A}\left(\mathcal{O}_{i}\right), \quad i=1,2,
$$

and hence $\left[\mathscr{A}_{\mathbf{g}}\left(\mathcal{O}_{1}\right), \mathscr{A}_{\mathbf{g}}\left(\mathcal{O}_{2}\right)\right]=\{0\}$.

b) $\mathscr{A}\left(\mathcal{O}_{2}\right) \subseteq \mathscr{A}\left(\mathcal{O}_{1}\right)+\mathscr{T}_{\mathrm{g}}$ is equivalent to $\mathscr{A}\left(\mathcal{O}_{2}\right)+\mathscr{T}_{\mathrm{g}} \subseteq \mathscr{A}\left(\mathbb{O}_{1}\right)+\mathscr{T}_{\mathrm{g}}$ and by $(2.5)$ and (2.6) equivalent to $\mathscr{A}_{\mathbf{g}}\left(\mathrm{O}_{2}\right) \subseteq \mathscr{A}_{\mathbf{g}}\left(\mathcal{O}_{1}\right)$. If $\operatorname{supp} f_{2} \subseteq \mathbb{C}_{2}$, then we can find (cf. Dimock $\left[3\right.$, Lemma A.3 and Theorem 4]) a $f_{1}, \operatorname{supp} f_{1} \subseteq \mathcal{O}_{1} \cap J\left(\operatorname{supp} f_{2}\right)$, and $G_{\mathrm{g}} f_{2}=G_{\mathrm{g}} f_{1}$. Hence, $G_{\mathbf{g}}\left(f_{2}-f_{1}\right)=0$ or $f_{2}-f_{1} \in \mathbf{K}_{\mathbf{g}}$, and we have $f_{2}^{\mathbf{g}}=f_{1}^{\mathbf{g}}$ or $W_{\mathbf{g}}\left(f_{2}^{\mathbf{g}}\right)$ $=W_{\mathrm{g}}\left(f_{1}^{\mathbf{g}}\right) \in \mathscr{A}\left(\mathbb{O}_{1}\right)$ and $\mathscr{A}_{\mathbf{g}}\left(\mathcal{O}_{2}\right) \cong \mathscr{A}_{\mathbf{g}}\left(\mathbb{O}_{1}\right)$.

The first flexible causality property (a) shows a connection between the metric tensor fields $\mathbf{g}$ on $\mathbb{R}^{4}$ and the maximal ideals $\mathscr{T}_{\mathrm{g}}$ of $\mathscr{A}$. This opens a possibility to use this relation in the definition of general causal structures in the next section. The primitive causality expresses in algebraic field theory the existence of a dynamical law. The second flexible causality property (b) shows that in $\mathscr{A}$ the ideals $\mathscr{T}_{\mathbf{g}}$ represent dynamical laws. As we considered only one type of dynamics, differing by the metrics $\mathbf{g}$, we can say that different maximal ideals $\mathscr{T}_{\mathbf{g}}$ are essentially characterized by the metrics. Only in this sense the maximal, two-sided ideals $\mathscr{T}_{\mathbf{g}}$ can be regarded as the analog of a metric tensor.

Now, we want to proof the general covariance of the net $\mathcal{O} \rightarrow \mathscr{A}(\mathcal{O})$. Let $\operatorname{Diff}\left(\mathbb{R}^{4}\right)$ be the group of all $C^{\infty}$-diffeomorphisms $\quad \kappa: \mathbb{R}^{4} \rightarrow \mathbb{R}^{4}, \quad \kappa(x)$ $=\left(\kappa^{0}(x), \kappa^{1}(x), \kappa^{2}(x), \kappa^{3}(x)\right)$. We define the linear transformations

$$
\kappa^{*}: f \rightarrow \kappa^{*} f, \kappa^{*} f(x):=f(\kappa(x)), \quad \text { and } \quad \kappa_{*}: f \rightarrow \kappa_{*} f, \kappa_{*}:=\left(\kappa^{-1}\right)^{*}=\left(\kappa^{*}\right)^{-1},
$$

of $\mathscr{D}$ onto itself. Let $\kappa(\mathcal{O})$ be the image of $\mathcal{O}$, then $\mathscr{D}(\kappa(\mathcal{O}))$ is the image of $\mathscr{D}(\mathcal{O})$ (test functions with support in $\mathcal{O}$ ) under $\kappa_{*}$. Furthermore (we use the same symbol for different mappings, but the meaning should become clear by the context),

$$
\begin{aligned}
\kappa^{*}: \mathbf{g} \rightarrow \kappa^{*} \mathbf{g}, \quad\left(\kappa^{*} \mathbf{g}\right)_{\mu \nu}(x):=\frac{\partial \kappa^{\alpha}(x)}{\partial x^{\mu}} \frac{\partial \kappa^{\beta}(x)}{\partial x^{v}} g_{\alpha \beta}(\kappa(x)), \\
\kappa_{*}: \mathbf{g} \rightarrow \kappa_{*} \mathbf{g}, \quad \kappa_{*}=\left(\kappa^{-1}\right)^{*}=\left(\kappa^{*}\right)^{-1},
\end{aligned}
$$

are one-to-one mappings of $\mathscr{\mathscr { G }}$ onto itself. To each $\kappa \in \operatorname{Diff}\left(\mathbb{R}^{4}\right)$ we want to associate an automorphism $\alpha_{\kappa}: \tilde{\mathscr{A}} \rightarrow \tilde{\mathscr{A}}$ which can be extended to all of $\mathscr{A}$. With the help of the flexible Weyl relation, each $A \in \tilde{\mathscr{A}}$ can be represented as $\mathscr{A}=\sum_{i=1}^{n} Z_{i} W\left(f_{i}\right)$ where the sections $Z_{i}: \mathbf{g} \rightarrow Z_{i}(\mathbf{g})$ are elements of the center of $\tilde{\mathscr{A}}$, i.e. $Z_{i}(\mathbf{g})$ is a multiple of the identity of $\mathscr{A}_{\mathrm{g}}$ and can be regarded as a complex number. On $\tilde{\mathscr{A}}$ we define

$$
\begin{gathered}
\alpha_{\kappa}(A):=\sum_{i=1}^{n} \alpha_{\kappa}\left(Z_{i}\right) \alpha_{\kappa}\left(W\left(f_{i}\right)\right), \\
\alpha_{\kappa}\left(W\left(f_{i}\right)\right):=W\left(\kappa_{*} f_{i}\right): \mathbf{g} \rightarrow W_{\mathbf{g}}\left(\left(\kappa_{*} f_{i}\right)^{\mathbf{g}}\right), \\
\alpha_{\kappa}\left(Z_{i}\right): \mathbf{g} \rightarrow Z_{i}\left(\kappa^{*} \mathbf{g}\right) .
\end{gathered}
$$

To proof that (2.7) defines an automorphism we need two lemmas. 
Lemma 2.2. $\sigma_{\mathbf{g}}\left(f^{\mathbf{g}}, h^{\mathbf{g}}\right)=\sigma_{\kappa^{*} \mathbf{g}}\left(\left(\kappa^{*} f\right)^{\kappa^{*} \mathbf{g}},\left(\kappa^{*} h\right)^{\kappa^{*} \mathbf{g}}\right)$ and $\kappa^{*}\left(f^{\mathbf{g}}\right)=\left(\kappa^{*} f\right)^{\kappa^{*} \mathbf{g}}$.

The proof of the above lemma follows by simple coordinate change technics which we shall omit.

Lemma 2.3. ${ }^{1}$

$$
i_{\left(\kappa^{*} \mathbf{g}, \mathbf{g}\right)}: W_{\mathbf{g}}\left(f^{\mathbf{g}}\right) \rightarrow W_{\kappa^{*} \mathbf{g}}\left(\left(\kappa^{*} f\right)^{\kappa^{*} \mathbf{g}}\right)
$$

and

$$
i_{\left(\kappa^{*} \mathbf{g}, \mathbf{g}\right)}\left(\sum_{i=1}^{n} \lambda_{i} W_{\mathbf{g}}\left(f_{i}^{\mathbf{g}}\right)\right):=\sum_{i=1}^{n} \lambda_{i} i_{\left(\kappa^{*} \mathbf{g}, \mathbf{g}\right)}\left(W_{\mathbf{g}}\left(f_{i}^{\mathbf{g}}\right)\right)
$$

defines an isomorphism of $\mathscr{A}_{\mathrm{g}}$ onto $\mathscr{A}_{\kappa^{*} \mathrm{~g}}$.

Proof. By the state (2.3) we have

$$
\omega\left(\sum_{i=1}^{n} \lambda_{i} W_{\mathbf{g}}\left(f^{\mathbf{g}}\right) \sum_{i=1}^{n} \lambda_{i}^{*} W_{\mathbf{g}}^{*}\left(f^{\mathbf{g}}\right)\right)=\sum_{i=1}^{n}\left|\lambda_{i}\right|^{2} .
$$

Hence, from $\sum_{i=1}^{n} \lambda_{i} W_{\mathbf{g}}\left(f_{i}^{\mathbf{g}}\right)=0$, all $f_{i}^{\mathbf{g}}$ different, we conclude $\lambda_{i}=0 \quad \forall i=1, \ldots, n$. Thus, $i_{\left(\kappa^{*} \mathbf{g}, \mathbf{g}\right)}$ is well defined on finite linear combinations. With help of Lemma 2.2 and the Weyl relation we conclude

$$
i_{\left(\kappa^{*} \mathbf{g} . \mathbf{g}\right)}\left(W_{\mathbf{g}}\left(f_{1}^{\mathbf{g}}\right) W_{\mathbf{g}}\left(f_{2}^{\mathbf{g}}\right)\right)=W_{\kappa^{*} \mathbf{g}}\left(\left(\kappa^{*} f_{1}\right)^{\kappa^{*} \mathbf{g}}\right) W_{\kappa^{*} \mathbf{g}}\left(\left(\kappa^{*} f_{2}\right)^{\kappa^{*} \mathbf{g}}\right) .
$$

As finite linear combinations of the $W_{\mathbf{g}}\left(f^{\mathbf{g}}\right)$ are dense, in norm, in $\mathscr{A}_{\mathbf{g}}$, we can extend $i_{\left(\kappa^{*} \mathrm{~g}, \mathrm{~g}\right)}$ uniquely to all of $\mathscr{A}_{\mathbf{g}}$.

Theorem 2.4. General Covariance. To every diffeomorphism $\kappa \in \operatorname{Diff}\left(\mathbb{R}^{4}\right),(2.7)$ defines an automorphism $\alpha_{\kappa}: \mathscr{A} \rightarrow \mathscr{A}$ that fullfills $\alpha_{\kappa}(\mathscr{A}(\mathcal{O}))=\mathscr{A}(\kappa(\mathcal{O}))$.

Proof. $\alpha_{\kappa}$ is well defined on $\tilde{\mathscr{A}}$, as by Lemma 2.2 and Lemma 2.3 we conclude

$$
\begin{aligned}
\sum_{i=1}^{n} Z_{i} W\left(f_{i}\right)=0 & \Leftrightarrow \sum Z_{i}(\mathbf{g}) W_{\mathbf{g}}\left(f_{i}^{\mathbf{g}}\right)=0 \quad \forall \mathbf{g} \in \mathscr{G}, \\
& \Leftrightarrow \sum Z_{i}\left(\kappa^{*} \mathbf{g}\right) W_{\kappa^{*} \mathbf{g}}\left(f_{i}^{\kappa^{*} \mathbf{g}}\right)=0 \quad \forall \mathbf{g} \in \mathscr{G}, \\
& \Leftrightarrow \sum Z_{i}\left(\kappa^{*} \mathbf{g}\right) W_{\mathbf{g}}\left(\left(\kappa_{*} f_{i}\right)^{\mathbf{g}}\right)=0 \quad \forall \mathbf{g} \in \mathscr{G}, \\
& \Leftrightarrow \sum_{i=1}^{n} \alpha_{\kappa}\left(Z_{i}\right) \alpha_{\kappa}\left(W\left(f_{i}\right)\right)=0 .
\end{aligned}
$$

Again, by Lemma 2.2 we can easily show that $\alpha_{\kappa}$ respects the flexible Weyl relation and all algebraic operations. Therefore, $\alpha_{\kappa}$ defines an automorphism on $\tilde{\mathscr{A}}$, which can uniquely be extended to all of $\mathscr{A} \cdot \mathscr{A}(0)$ is generated by all those $W(f)$ with $\operatorname{supp} f$ in $\mathcal{O}$. With $\alpha_{\kappa}(W(f))=W\left(\kappa_{*} f\right)$ and $\kappa_{*}(\mathscr{D}(\mathcal{O}))=\mathscr{D}(\kappa(\mathcal{O}))$ we finally get $\alpha_{\kappa}(\mathscr{A}(\mathcal{O}))=\mathscr{A}(\kappa(\mathcal{O}))$.

Because of this theorem and the validity of the flexible Weyl relation, we also call $\mathscr{A}$ the generally covariant Weyl algebra.

${ }^{1}$ Compare the related result of Dimock [3, Theorem 4] 
We can interprete the automorphism $\alpha_{\kappa}$ in two manners:

I) If $A \in \mathscr{A}$ represents a measurement, then $\alpha_{\kappa}(A)$ may be a completely different described measurement, where the description of $A$ is altered by the diffeomorphism $\kappa . W(f)$ will be replaced by $W\left(\kappa^{*} f\right)$. The laboratory of the observer stays in the same state of motion or the observer stays in the same reference frame.

II) $\kappa$ defines a transition to another reference frame (laboratory) of the observer. Now $\alpha_{\kappa}(A)$ represents the same measurement as $A$. The physical effect is the alteration of expectation values of states. (With this idea of general covariance, Fredenhagen and Haag [8] calculated the response of an accelerated detector.)

We want to add some remarks on states of $\mathscr{A}$. If a state $\omega$ fullfills $\omega\left(\mathscr{T}_{\mathbf{g}}\right)=\{0\}$, then all measurements reduce to those of the net given by $\mathcal{O} \rightarrow \mathscr{A}(\mathcal{O}) / \mathscr{T}_{\mathrm{g}}$ which is no more generally covariant and which has a fixed dynamical and causal structure. Therefore, a state may break the symmetry. By this effect gravitation comes into play and $\omega$ may be interpreted as a state on a fixed gravitational background. A state $\omega$ which fullfills $\omega\left(\bigcap_{i} \mathscr{T}_{\mathbf{g}_{2}}\right)=\{0\}$ is a mixture of states $\omega_{i}$ with $\omega_{i}\left(\mathscr{T}_{\mathbf{g}_{2}}\right)=\{0\}$ and, therefore, a mixture over different gravitational backgrounds $\mathrm{g}$. These are states with classical gravitational fluctuations which perhaps may serve as a first approximation to states of a real quantum gravitational field. By the existence of these states, which cannot be ruled out at once by physical arguments, the presented scheme is more general than that given by Dimock [3].

\section{Generalizations}

In Sect. 2 the inputs were the dynamical laws respectively the metric fields, which define the causal and dynamical structures. By Theorem 2.1 these could be expressed by the flexible causality relations. We now want to go the opposite way. Starting with a net $\mathcal{O} \rightarrow \mathscr{A}(\mathcal{C})$ of $C^{*}$-algebras defined (for simplicity again) on $\mathbb{R}^{4}$, we shall define causal and dynamical structures ${ }^{2}$ by special ideals of the algebra $\mathscr{A}$ which is associated with $\mathbb{R}^{4}$.

The first flexible causality property of Theorem 2.1 motivates the definition of causally independent sets.

Definition 3.1. If $\mathscr{T}$ is an ideal (in the following, every ideal is two-sided and closed in norm), then two sets $\mathscr{U}_{1}, \mathscr{U}_{2} \subseteq \mathbb{R}^{4}$ are defined to be causally independent with respect to $\mathscr{T}$, if there are regions $\mathscr{O}_{1}, \mathfrak{O}_{2} \subseteq \mathbb{R}^{4}$, such that $\mathscr{U}_{1} \subseteq \mathcal{O}_{1}, \mathscr{U}_{2} \subseteq \mathcal{O}_{2}$, and $\left[\mathscr{A}\left(\mathcal{O}_{1}\right), \mathscr{A}\left(\mathcal{O}_{2}\right)\right] \subseteq \mathscr{T}$. A continuous curve of $\mathbb{R}^{4}$ will be called $\mathscr{T}$-causal curve, if no two points of $\mathbb{R}^{4}$ are causally independent with respect to $\mathscr{T}$.

The second flexible causality property of Theorem 2.1 reflects the presence of dynamical laws. For its general formulation we need a definition for the domain of dependent of a region.

Definition 3.2. For a region $\mathcal{O} \subset \mathbb{R}^{4}$ we define $D_{\mathscr{T}}(\mathcal{O}):=\left\{p \in \mathbb{R}^{4} \mid\right.$ every (non extendible) $\mathscr{T}$-causal curve through $p$ intersects $\left.\mathcal{O}\right\}$ to be the domain of dependence of $\mathcal{O}$.

${ }^{2}$ The following definitions differ slightly from that given in [1] 
Definition 3.3. Let $\mathscr{T}$ be an ideal in $\mathscr{A}$. If for each pair of regions $\mathcal{O}_{1}, \mathcal{O}_{2}$ of $\mathbb{R}^{4}$ we have

$$
\mathcal{O}_{2} \subseteq D_{\mathscr{T}}\left(\mathcal{O}_{1}\right) \Rightarrow \mathscr{A}\left(\mathcal{O}_{2}\right) \subseteq \mathscr{A}\left(\mathbb{U}_{1}\right)+\mathscr{T}
$$

then $\mathscr{T}$ is called hyperbolic.

For a net $\mathcal{O} \rightarrow \mathscr{A}(\mathcal{O})$ to have well defined causal and dynamical structures, $\mathscr{A}$ should possess hyperbolic ideals. But there is yet no general answer to the question, which ideals of $\mathscr{A}$ should be hyperbolic.

The hyperbolic ideals seem to be proper candidates for algebraic pendants of dynamical equations or Lagrangians and commutation relations of usual field theories. A net with hyperbolic ideals should show many essential properties of a quantum field theory on a general space-time.

As pointed out, by the presented frame we can get no quantum gravity. We believe that in an algebraic quantum gravity - if at all possible - there should be no two-sided ideals, but structures which represent ideals in some approximate sense to formulate approximate causal and dynamical structures. Therefore, the study of the above structures (which should be confirmed or corrected by more examples) by purely algebraic methods may even lead to further insight into real quantum gravity.

Acknowledgements. It is a pleasure to thank Prof. Dr. R. Haag and Prof. Dr. K. Fredenhagen for many helpful and stimulating discussions.

\section{References}

1. Bannier, U.: Allgemein kovariante algebraische Quantenfeldtheorie und Rekonstruktion von Raum-Zeit. Thesis, Hamburg 1987

2. Brattelli, O., Robinson, D.W.: Operator algebras and quantum statistical mechanics, Vol. II. Berlin, Heidelberg, New York: Springer 1981

3. Dimock, J.: Algebras of local observables on a manifold. Commun. Math. Phys. 77, 219 (1980)

4. Dixmier, J.: $C^{*}$-algebras. Amsterdam, New York, Oxford: North-Holland 1977

5. Dubois-Violette, M.: A generalization of the classical moment problem on *-algebras with application to relativistic quantum theory. I. Commun. Math. Phys. 43, 225 (1975)

6. Dyson, F.J.: Missed opportunities. Bull. Am. Math. Soc. 78, 635 (1972)

7. Ekstein, H.: Presymmetry. II. Phys. Rev. 184, 1315 (1969)

8. Fredenhagen, K., Haag, R.: Generally covariant quantum field theory and scaling limits. Commun. Math. Phys. 108, 91 (1987)

9. Friedlander, F.G.: The wave equation on a curved space-time. Cambridge, London, New York, Melbourne: Cambridge University Press 1975

10. Haag, R., Kastler, D.: An algebraic approach to quantum field theory. J. Math. Phys. 5, 848 (1964)

Communicated by A. Jaffe

Received February 24, 1988 